\title{
Exploiting Zinc Oxide Re-Emission to Fabricate
}

\section{Periodic Arrays}

Michael J. Coutts, Hadi M. Zareie ${ }^{1}$, Michael B. Cortie, Matthew R. Phillips, Richard Wuhrer and Andrew M. McDonagh

Institute for Nanoscale Technology, University of Technology Sydney, P.O. Box 123, Broadway NSW 2007, Australia

Email: hadi.zareie@uts.edu.au

Telephone: +61-(0)2-9514-8330

Fax: +61-(0)2-9514-1703

Keywords: Re-emission, zinc oxide nano-structures, nanosphere lithography, solution growth, nanosandwiches

\footnotetext{
Abstract: The synthesis of hexagonal ring-shaped structures of zinc oxide using nanosphere lithography and metal / metal oxide sputtering is demonstrated. This synthesis exploits the surface reemission of zinc oxide to deposit material in regions lying out of the line-of-sight of the sputtering source. These rings can nucleate the hydrothermal growth of zinc oxide crystals. Control over the growth could be exercised by varying growth solution concentration or temperature, or by applying an external potential.
} 


\section{INTRODUCTION}

Zinc oxide nano and microstructures are attracting an increasing amount of interest due largely to their interesting optoelectronic properties and high emission efficiency for potential applications such as lighting(1) and photodetectors(2) as well as low-loss surface acoustic wave (SAW) filters(3), sensor arrays (4), transparent conductive oxides(5) and UV photoemitters(6). Much research has focused on the synthesis of nanostructures using techniques such as electrodeposition, $(5,7)$ epitaxial thin film growth,(2, 8), high temperature vapor deposition for nanowires, $(9,10)$ and solution deposition of nanostructures including nanowires(11-15), nanotubes(7), and "nanopine-trees."(16) The technique of nanosphere lithography (NSL) uses mono- or bi-layers of close-packed latex spheres as a template to produce relatively large area arrays of nanoscale structures $(14,17-23)$. The spheres act as a mask and materials are deposited through the voids between the spheres onto the substrate using evaporation or sputtering processes $(17,19)$. Generally, NSL produces regular arrays of triangular structures but here we show that remarkable hexagonal ring-shaped structures of zinc oxide can also be fabricated using NSL and metal / metal oxide sputtering. This is achieved by exploiting the re-emission (24) of the zinc oxide from the surface to produce ring-shaped structures lying out of the line-of-sight of the sputtering source. Our rings can be further developed by immersion of the templates into an aqueous growth solution from which zinc oxide can precipitate (7, 11-16). These findings provide a straightforward method to produce arrays of micron-sized rings of this material.

\section{RESULTS AND DISCUSSION}
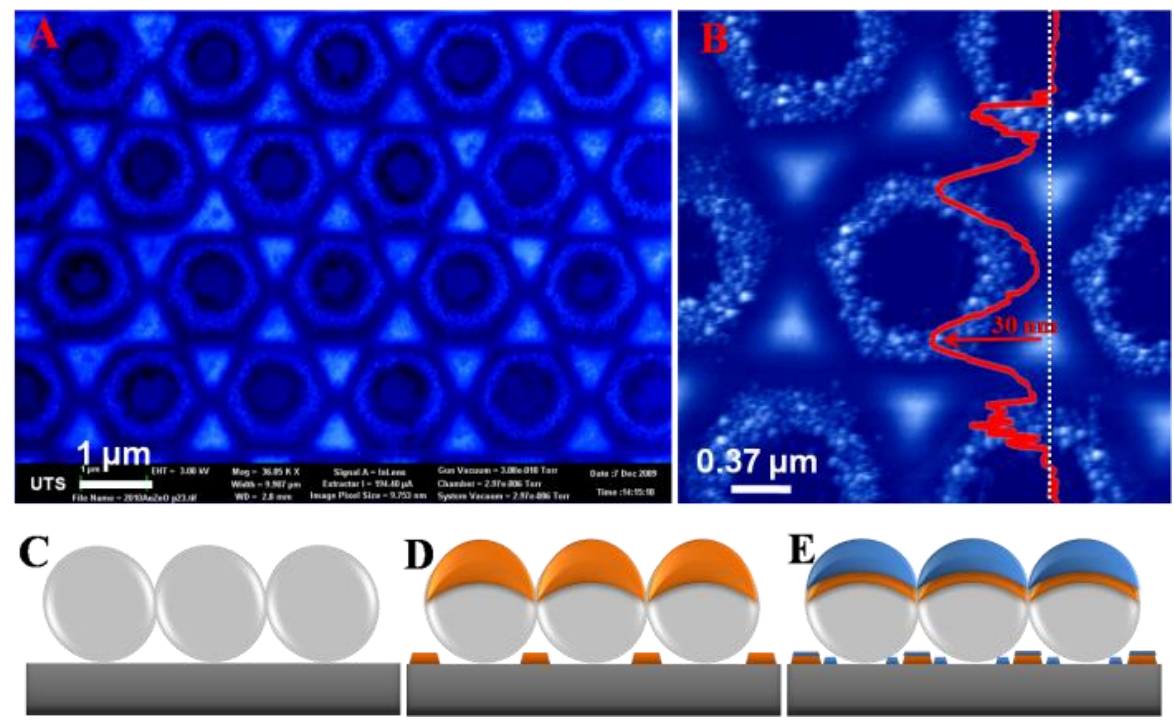

FIGURE 1. (A) SEM micrograph of triangular pyramids and zinc oxide rings that result from the metal/metal oxide sputtering of $20 \mathrm{~nm}$ of gold followed by $10 \mathrm{~nm}$ of zinc oxide (after removal of the latex spheres). (B) AFM image of the structures in A: with corresponding line profile (C-E) Schematic showing the procedure to prepare the new structures 
FIGURE 1A shows a scanning electron microscope (SEM) image of an array of the new structures. The array consists of two shapes: compact composite triangles made from a layer of zinc oxide on top of a layer of gold, and loosely packed granular rings of zinc oxide. The rings have a degree of hexagonal symmetry. Remarkably, the rings were deposited in regions that were shaded by the latex spheres. The zinc oxide particles are more densely packed at the outer edges of the hexagonal rings and become more isolated toward the inner region of the ring (see Supporting Information FIGURE S1). The edges of the triangles are $\sim 800 \mathrm{~nm}$ in length while the hexagonal rings are $\sim 1.3 \mu \mathrm{m}$ from face to face as indicated in the supporting information FIGURE S1. The arrays of the nanostructures were prepared using nanosphere lithography $(17,19)$ and DC/RF magnetron sputtering, as illustrated in FIGURE 1C-E. An aqueous suspension of polystyrene spheres (diameter $1.58 \mu \mathrm{m}$ ) was spin-coated onto a cleaned silicon substrate. The spheres exhibit hexagonal close packing across reasonably large areas of the surface (FIGURE S2). Gold was then deposited by DC magnetron sputtering, FIGURE 1D, followed by zinc oxide deposited with RF sputtering, FIGURE 1E. The latex spheres were then removed by thorough sonication and rinsing in dichloromethane.

Importantly, a gold layer is critical to ring formation; only triangular structures of zinc oxide were formed when no gold was deposited first (FIGURE S3). The atomic force microscope (AFM) data shown in FIGURE 1B reveals the topography of a $3 \mu \mathrm{m}$ x $3 \mu \mathrm{m}$ region of an array. A line profile shows that the height of the triangular structures is $\sim 30 \mathrm{~nm}$, which is in agreement with $20 \mathrm{~nm}$ of gold and 10 $\mathrm{nm}$ of zinc oxide as measured by a quartz crystal microbalance during the deposition process. The hexagonal rings have a height of $\sim 15 \mathrm{~nm}$. Energy dispersive X-ray spectroscopy data (FIGURE S4) confirm that the rings contain zinc and oxygen while the triangular structures contain gold, zinc and oxygen. Carbon was detected across each of these regions and was found to be ubiquitous in all samples investigated.

The dimensions of the hexagonal ring structures indicate that they form in a region that was shaded by the latex spheres from direct "line-of-sight" of the sputter target. To deposit in these shaded areas the sputtered zinc oxide must either have been deflected from its trajectory in-flight or have been re-emitted from the surface of the triangles. In fact, re-emission of zinc oxide is well-known with re-sputtering coefficients for zinc oxide reported to be as high as 0.3 (25). Under appropriate conditions and geometries, significant quantities of material can be deposited in areas not in line-of-site of the source $(24,26-31)$ and the deposits are reportedly loosely packed (25), as is the case in the current work. However, re-emission from the surface is not sufficient on its own to explain the ring-shaped deposits of zinc oxide described here because the rings were not formed when the gold under-layer was absent. Clearly, the gold plays a vital role in the process. 
It is known that zinc oxide and gold can form a Schottky diode(32), with the oxide serving as an ntype semiconductor (33). Therefore, negative charge may accumulate on the zinc oxide that overlies the gold in the triangles, with the charge induced by electric fields and/or charged species within the sputter chamber. Once sufficient charge had developed, negatively charged zinc oxide species may be deflected away from the triangles. However, deflection of incoming charged species is unlikely as their concentrations are reportedly only a few percent $(34,35)$. Furthermore, the kinetic energy of the incoming zinc oxide is high, as evidenced by the compact nature of the zinc oxide on the triangle. On the other hand, re-emission of zinc oxide from a deposit is an effective process and is known to be enhanced by negatively charged surfaces (24) as they can attract positively charged argon ions. We propose therefore, that it is the diodic characteristics of the composite zinc oxide/gold triangles that are responsible for the directed deposition of the re-emitted zinc oxide to form the unusual hexagonal ring structures.

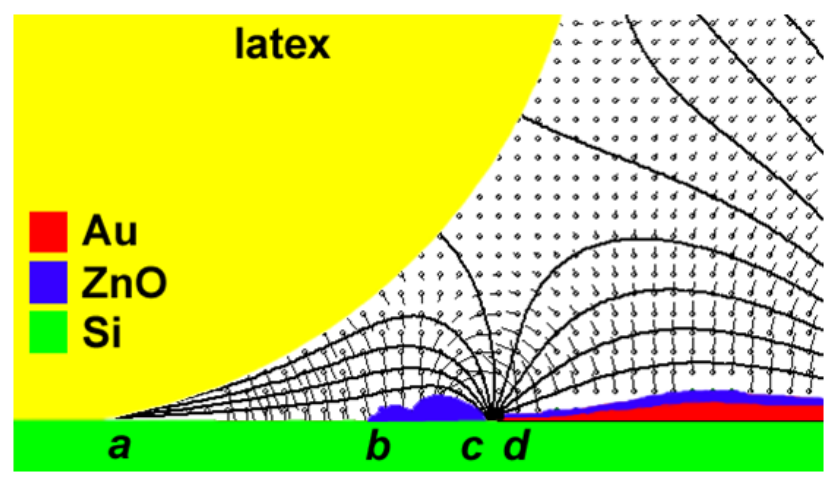

FIGURE 2. Proposed mechanism for the formation of the zinc oxide rings. Distribution of electric equipotentials (solid lines) and direction of field lines (vectors) for the case where the triangle has accumulated a negative potential relative to the silicon substrate (the latex sphere is treated as an insulator in this simulation). The drawing is to scale and uses the experimental AFM topography in the calculation. The zinc oxide is the blue phase.

Support for this mechanism may be deduced from the location of the re-emitted deposit. FIGURE 2 shows the results of modeling the electric field in the vicinity of the substrate using the Poisson equation. In the simulation shown, a charge of $0 \mathrm{~V}$ was allocated to the latex sphere, $-1 \mathrm{~V}$ to the triangle, and $0 \mathrm{~V}$ to the silicon substrate. Simulations for other situations, and for the case in which the polymer sphere is considered to be an insulator, produced similar results; the only important criterion for ring formation is that the triangles should be at a more negative potential than the surrounding material. In all cases, any negatively charged zinc oxide species re-emitted from the triangular surface can be propelled along the electric field lines into the space lying under the polymer sphere in the region between points $b$ and $c$. However, very few species are likely to settle in the zone between points $c$ and $d$ because the 
electric field lines in that region of the sample are directed parallel to the surface of the silicon substrate. Furthermore, few species will travel to the region between points $a$ and $b$ because the electric field lines in that regions point back to the undersurface of the latex sphere. It can be seen by following the field vectors that little or no charged, remitted material would deposit in this region. The regions between points $a$ and $b$, and between $c$ and $d$, correspond to the regions free of zinc oxide observed in experiments, while the region $b-c$ corresponds to the experimentally observed zinc oxide ring.

The absence of a ring in the case where no gold layer was present is readily explained as any charge on the zinc oxide triangles drains through the silicon substrate to ground, resulting in no local electric fields. Although re-emission of zinc oxide would still occur, very little material would be preferentially deflected into the region $b-c$ because of the absence of a local electric field.

The gold itself is also likely to form a Schottky diode with the silicon (36) and in the case of a p-type silicon substrate this would preferentially channel negative charge from the substrate to the gold and from there through the gold/zinc oxide junction into the overlying zinc oxide. In contrast, use of an ntype silicon would channel holes into the gold and this would in turn reduce the rectification on the gold/zinc oxide junction (by promoting a greater reverse current of electrons from zinc oxide to gold) and hence reduce the proposed negative charge on the zinc oxide. Experimentally, we found that the ring formations could be produced on either n- or p-type silicon, FIGURE S5, but the density of those on ntype silicon was lower than on p-type, in agreement with the proposed mechanism.
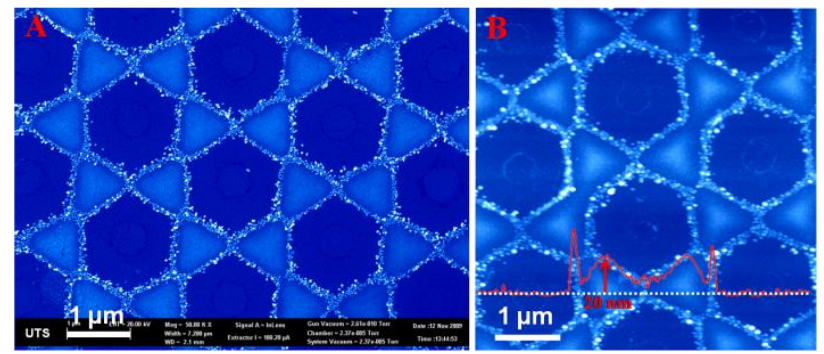

FIGURE 3. (A) SEM micrograph of arrays fabricated by deposition of platinum (thickness $20 \mathrm{~nm}$ ) and zinc oxide (thickness $10 \mathrm{~nm}$ ). (B) AFM data images of the sample shown in panel A.

An interesting result was observed when platinum was deposited rather than gold, FIGURE 3. The AFM data reveal that the triangular pyramids formed are broader and flatter when compared to gold. Zinc oxide, however, does not deposit over as broad an area, hence uncoated regions of positively charged or grounded platinum are exposed when the zinc oxide re-emits. Any negatively charged zinc oxide species will be attracted to these regions, as illustrated in FIGURE 3. Experiments using silver yielded very similar structures to those using gold (see Supporting Information, FIGURE S6). The deposited silver triangular pyramids more closely resembled those fabricated using gold rather than those made from platinum. Silver / zinc oxide heterojunctions have Schottky barrier heights similar to 
those of gold / zinc oxide (32) with both lying between $0.65-0.70 \mathrm{eV}$. This suggests that both the metal / metal oxide Schottky barrier and deposition geometry are important factors in the subsequent zinc oxide ring formation.

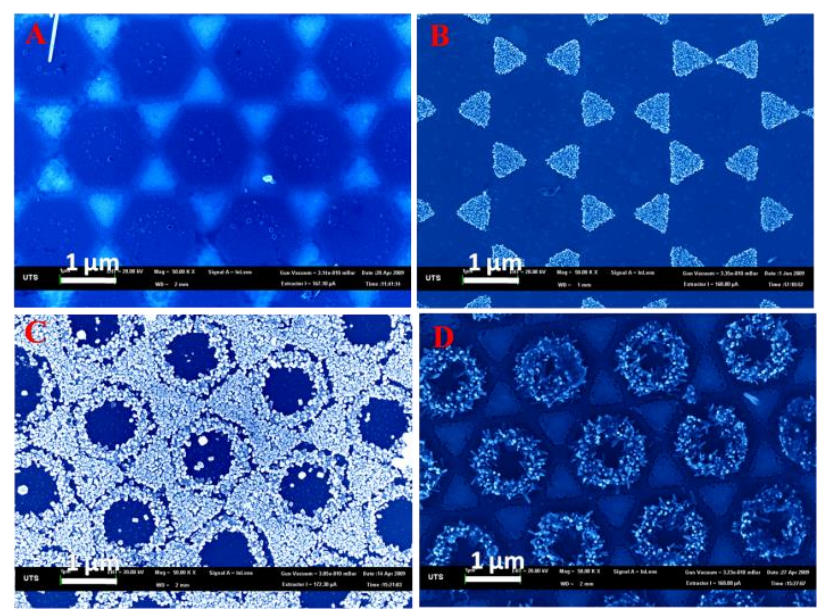

FIGURE 4. SEM micrographs of arrays fabricated by deposition of zinc oxide from zinc nitrate solutions onto templates with various compositions. Samples were all incubated for $3 \mathrm{hrs}$ at $90{ }^{\circ} \mathrm{C}$ in aqueous solution containing zinc nitrate $(10 \mathrm{mM})$ and hexamine $(10 \mathrm{mM})$. (A) Gold (20 nm). (B) Zinc oxide (10 nm). (C) Gold (20 nm) / zinc oxide (10 nm). (D) Gold (20 nm) / zinc oxide (20 nm) / gold (20 $\mathrm{nm}$ ). Higher magnification SEM micrographs of (C) and (D) are available in the supporting information FIGURE S7.

The new nanostructured arrays were investigated as templates to seed the hydrothermal growth of zinc oxide. The templates were immersed in aqueous solutions of zinc nitrate and hexamine (14). Gold / zinc oxide / gold sandwich-type array structures were prepared with different thicknesses of gold and zinc oxide to investigate their role in the subsequent zinc oxide growth. The reaction temperature, reactant concentration and template array structure were each found to have a significant influence on the type of zinc oxide structures that formed. FIGURE 4 shows the results of zinc oxide growth experiments using various template structures. Gold-only triangles, zinc oxide-only triangles, triangles consisting of gold capped with zinc oxide, as well as gold / zinc oxide / gold sandwiches with the associated hexagonal ring structures were investigated. The growth conditions were identical for each substrate used and, importantly, during immersion in the growth solution the substrates were placed face-down to minimize settling of precipitates onto the surface. FIGURE 4A reveals that almost no zinc oxide growth occurs when gold-only arrays are used. This is in contrast to some reported experiments performed with the substrate facing up where growth is presumably seeded by settled precipitates (15) and also in contrast to physical vapour growth methods where only gold is required to catalyze growth but at significantly elevated temperatures (37). FIGURE 4B shows that limited growth occurs when zinc oxide templates 
are used without gold and that growth occurs only directly on top of the triangles. Significantly more zinc oxide growth is seen in FIGURE 4C both on top of and around the edges of the triangle structures, which consist of $10 \mathrm{~nm}$ of zinc oxide on top of $20 \mathrm{~nm}$ of gold. It is apparent that the NSL-deposited zinc oxide seeds the subsequent growth but the combination of gold and zinc oxide in the triangles significantly accelerates zinc oxide growth. Interestingly, as well as growth on the triangular template surfaces, the ring-shaped zinc oxide deposits formed in the hexagonal voids between the triangles also facilitated growth. FIGURE 4D shows zinc oxide growth using gold / zinc oxide / gold sandwich-type templates, here the top layer of gold caps the triangular pyramid limiting zinc oxide growth to the ring shaped regions.

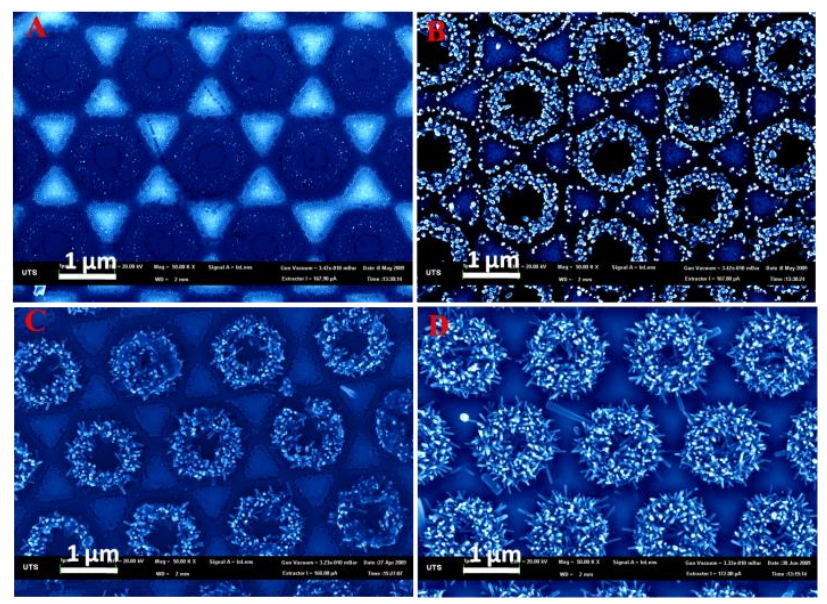

FIGURE 5. SEM micrographs of arrays fabricated by deposition of zinc oxide from zinc nitrate solutions of various concentrations onto templates consisting of gold $(20 \mathrm{~nm}) /$ zinc oxide $(20 \mathrm{~nm}) /$ gold $\left(20 \mathrm{~nm}\right.$ ) and incubated at $90{ }^{\circ} \mathrm{C}$ for 3 hrs. Concentrations of zinc nitrate are; (A) $0.1 \mathrm{mM}$. (B) $1 \mathrm{mM}$. (C) $10 \mathrm{mM}$. (D) $0.1 \mathrm{M}$. A higher magnification SEM micrograph of (D) is available in the supporting information FIGURE S8.

Increasing the concentration of zinc nitrate and hexamine in the growth solution resulted in amplified zinc oxide growth on the templates. FIGURE 5(A-D) shows examples of gold (20 nm) / zinc oxide (20 $\mathrm{nm})$ / gold $(20 \mathrm{~nm})$ templates immersed in zinc nitrate and hexamine solutions of concentrations ranging from $0.1 \mathrm{mM}$ to $0.1 \mathrm{M}$. As well as the amplification in coverage, higher concentrations resulted in rodlike structures with increasing diameters. These results are in agreement with those published recently by Xu et al. (38)

The template growth of zinc oxide on samples incubated at 80,90 and $150{ }^{\circ} \mathrm{C}$ was also examined. Amplified growth was observed with increasing temperature (FIGURE S9) in agreement with previous studies (38-40). At $150{ }^{\circ} \mathrm{C}$, many of the zinc oxide rods formed tube-shaped structures with clean, hexagonal facets (FIGURE S10). 


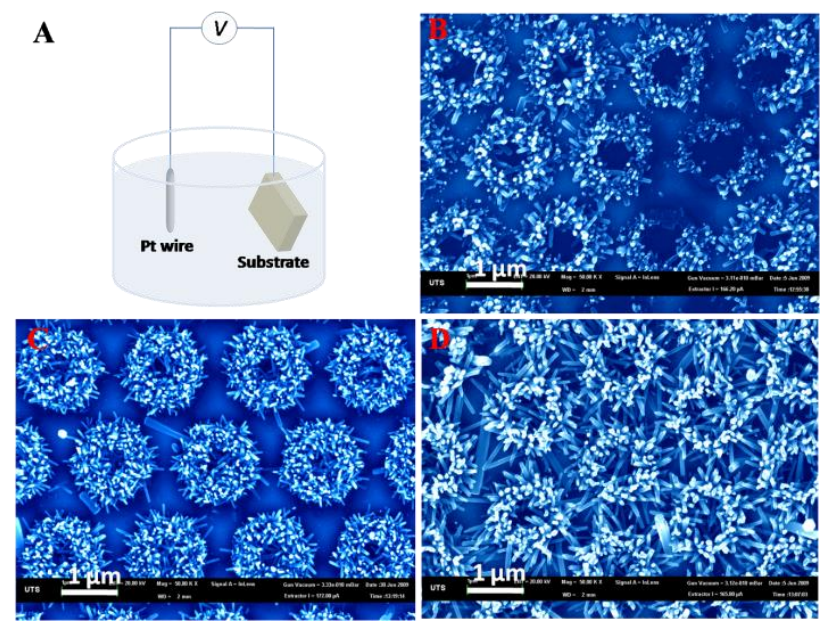

FIGURE 6. (A) Schematic illustration showing the apparatus used to apply bias during zinc oxide growth. (B-D) SEM micrographs of arrays fabricated by growth of zinc oxide from zinc nitrate solution on templates consisting of gold $(20 \mathrm{~nm}) /$ zinc oxide $(20 \mathrm{~nm}) /$ gold $(20 \mathrm{~nm})$ at $90^{\circ} \mathrm{C}$ for $3 \mathrm{hrs}$ with various applied potentials; (B) $-0.5 \mathrm{~V}$. (C) $0 \mathrm{~V}$. (D) $+0.5 \mathrm{~V}$ potentials.

An applied bias has been reported to affect the rate of growth of zinc oxide structures, which deposit on the cathode $(13,41)$. To examine the effect of applied bias on the zinc oxide growth templates, the experimental set up shown in FIGURE 6A was assembled. Substrates were immersed in a growth solution and a potential of either $0,+0.5$ or $-0.5 \mathrm{~V}$ (with respect to the counter-electrode, where $+0.5 \mathrm{~V}$ indicates that the substrate was cathodic) was applied. The SEM micrographs shown in FIGURE 6 reveal that applying a potential to the samples during growth had a significant impact on zinc oxide growth. An applied potential of $-0.5 \mathrm{~V}$ diminished growth while a potential of $+0.5 \mathrm{~V}$ resulted in amplified growth relative to the sample with $0 \mathrm{~V}$ applied. This indicates that, in this environment, zinc can be conveyed to the surface of a growing crystal in some positively charged complex.

Cathodoluminescence (CL) measurements confirmed the composition and highlighted some local stress properties $(42,43)$ of some zinc oxide nanostructures grown by templated solution precipitation. $\mathrm{CL}$ spectra from the ring-shaped $\mathrm{ZnO}$ deposits reveal a weak excitonic ultra-violet peak located at $3.22 \mathrm{eV}(385 \mathrm{~nm})$ along with a slightly stronger green luminescence peak at around $2.5 \mathrm{eV}(\sim 495 \mathrm{~nm})$, which is generally attributed to oxygen related surface and bulk recombination centres in zinc oxide (44). A typical CL spectrum exhibited by the zinc oxide nanorods is shown in FIGURE S11. The CL emission is comprised of a strong red luminescence peak centred at $1.6 \mathrm{eV}(\sim 775 \mathrm{~nm})$ and a weak excitonic near band edge emission at $3.22 \mathrm{eV}$. The red luminescence peak has been assigned to a donoracceptor pair transition between neutral effective mass donors and $\mathrm{V}_{\mathrm{Zn}}{ }^{-}$acceptors (45). CL imaging (FIGURE S11) reveals that the red luminescence increases from the base of the nanowire and is strongest at its tip, suggesting that the donor-acceptor pair concentration is highest at the growth ends. 
These data indicate that the template structure as well as the $\mathrm{ZnO}$ growth times and temperatures have a significant effect on the type of native defect and their distribution within the $\mathrm{ZnO}$ nano-structures.

\section{CONCLUSION}

In summary, we have prepared arrays of gold / zinc oxide triangles and zinc oxide rings on a silicon substrate using nanosphere lithography. The rings, which possess a degree of hexagonal symmetry, are evidently formed by the re-emission of $\mathrm{ZnO}$ from the upper surfaces of the triangles. This phenomenon required the presence of an underlying layer of $\mathrm{Au}$ on the base of the triangle and was absent if the zinc oxide was deposited on bare silicon. The rings of zinc oxide can, in turn, serve as nuclei for the subsequent development of the zinc oxide crystals from an aqueous growth solution. Further control of the growth could be exercised by varying the growth solution concentration or temperature, or by applying an external potential.

\section{EXPERIMENTAL}

General. Silicon (p-type; 100, ProSciTech. n-type; 111, ProSciTech) latex spheres (10 wt \% solution in water, Bang laboratories, $1.58 \mu \mathrm{m})$, triton-X100 (Aldrich), methanol (Chem Supply), gold (AGR Matthey), zinc oxide (Williams Advanced Materials), zinc nitrate (Ajax), hexamine (BDH), ethanol (Chem Supply), dichloromethane (Lab scan) were all used as received. Spin coating was performed on a Headway Research spin coater. Gold and zinc oxide were deposited in an Edwards E306 deposition chamber equipped with a DC/RF magnetron sputter system with an argon partial pressure of $3 \times 10^{-4}$ Torr. Deposition rate and film thickness were monitored with a Maxtek TM-200 quartz crystal microbalance.

Nanosphere lithography. Gold / zinc oxide arrays were synthesized using a modified literature procedure.(21) Silicon substrates (approx $1 \mathrm{~cm}^{2}$ ) where immersed in piranha solution (3:1 $\mathrm{H}_{2} \mathrm{SO}_{4}: 33 \% \mathrm{H}_{2} \mathrm{O}_{2}$ : CAUTION) for $0.5 \mathrm{~h}$ and rinsed thoroughly with MilliQ water rendering the surface hydrophilic. Latex sphere solution $(400 \mu \mathrm{L})$ was added to $100 \mu \mathrm{L}$ of a solution of triton-X in methanol (1:400 vol/vol). Then, $20 \mu \mathrm{L}$ of the resultant suspension was dropped onto the silicon and then spun at 2000 r.p.m. for 40 s. The substrate was then transferred to a vacuum deposition chamber $\sim 10^{-6}$ Torr $\left(\sim 1 \times 10^{-4} \mathrm{~Pa}\right)$ base pressure. Argon was leaked into the chamber to a partial pressure of $3 \times 10^{-4}$ Torr 
$\left(4 \times 10^{-2} \mathrm{~Pa}\right)$ and gold was deposited with an Advanced Energy MDX 500 power supply (0.017 A, $\left.20 \mathrm{~V}\right)$.

Zinc oxide was deposited with an Advanced Energy RFX-600 power supply (50W). The distance between the sample and target was $15 \mathrm{~cm}$. After deposition, the substrates were immersed in dichloromethane, sonicated for $2 \mathrm{~min}$ and then thoroughly rinsed with dichloromethane and dried under a stream of nitrogen gas.

Zinc oxide growth experiments

Samples were heated for $3 \mathrm{~h}$ in a sealed tube containing $20 \mathrm{~mL}$ of a solution of zinc nitrate and hexamine (1:1 stoichiometry). Concentrations were varied from $0.1 \mathrm{mM}$ up to $0.1 \mathrm{M}$. Important: samples were placed with the template side face down to minimize $\mathrm{ZnO}$ deposition from precipitates. After $3 \mathrm{~h}$, samples were rinsed thoroughly with ethanol.

AFM Imaging. A digital multimode Nanoscope III scanning force microscope was used. All imaging was conducted in the tapping mode, with $512 \times 512$ data acquisitions at a scan speed of $0.6 \mathrm{~Hz}$ at room temperature in air. Oxide-sharpened silicon nitride tips with integrated cantilevers with a nominal spring constant of $0.38 \mathrm{~N} / \mathrm{m}$ were used.

SEM Imaging / EDS measurements. SEM images were obtained using a LEO Supra 55 VP microscope (Zeiss) equipped with an in-lens secondary-electron detector. Images were manipulated using scanning probe image processor (SPIP) software, and contrast was enhanced using Adobe Photoshop to allow discrimination of fine detail. EDS spectra were obtained with a Oxford instrument (INCA X-sight) Xray detector.

\section{Cathodoluminescence experiments}

Cathodoluminescence (CL) microscopy and spectroscopy was performed using a FEI Quanta 200 SEM equipped with a Gatan MonoCL3 system. All CL spectra were corrected for the detection response of the system.

Acknowledgment We thank the Australian Research Council (DP0877539) for financial support and the Microstructural Analysis Unit, UTS. 
Supporting Information Available: SEM images, EDS spectra, Cathodoluminesence measurements. This material is available free of charge via the internet at http://pubs.acs.org.

\section{REFERENCES AND NOTES}

1. Saito, N.; Haneda, H.; Sekiguchi, T.; Ohashi, N.; Sakaguchi, I.; Koumoto, K., Advanced Materials 2002, 14, (6), 418-421.

2. Lee, J. Y.; Choi, Y. S.; Kim, J. H.; Park, M. O.; Im, S., Thin Solid Films 2002, 403-404, 553557.

3. Emanetoglu, N. W.; Gorla, C.; Liu, Y.; Liang, S.; Lu, Y., Materials Science in Semiconductor Processing 1999, 2, (3), 247-252.

4. Kumar, N.; Dorfman, A.; Hahm, J., Nanotechnology 2006, 17, (12), 2875-2881.

5. Rousset, J.; Saucedo, E.; Lincot, D., Chemistry of Materials 2009, 21, (3), 534-540.

6. Chen, Y.; Bagnall, D.; Yao, T., Materials Science and Engineering B 2000, 75, (2-3), 190-198.

7. $\quad$ Elias, J.; Tena-Zaera, R.; Wang, G.-Y.; LeÌ $\square$ vy-CleÌ $\square$ ment, C., Chemistry of Materials 2008, 20, (21), 6633-6637.

8. $\quad$ Liang, S.; Sheng, H.; Liu, Y.; Huo, Z.; Lu, Y.; Shen, H., Journal of Crystal Growth 2001, 225, (2-4), 110-113.

9. Wang, X.; Li, G.; Chen, T.; Yang, M.; Zhang, Z.; Wu, T.; Chen, H., Nano Lett. 2008, 8, (9), 2643-2647.

10. Hwang, W.; Choi, J.-H.; Kim, T. H.; Sung, J.; Myoung, J.-M.; Choi, D.-G.; Sohn, B.-H.; Lee, S. S.; Kim, D. H.; Park, C., Chemistry of Materials 2008, 20, (19), 6041-6047.

11. Liu, D. F.; Xiang, Y. J.; Wu, X. C.; Zhang, Z. X.; Liu, L. F.; Song, L.; Zhao, X. W.; Luo, S. D.; Ma, W. J.; Shen, J.; Zhou, W. Y.; Wang, G.; Wang, C. Y.; Xie, S. S., Nano Letters 2006, 6, (10), 23752378 .

12. Kim, Y. J.; Lee, C. H.; Hong, Y. J.; Yi, G. C.; Kim, S. S.; Cheong, H., Applied Physics Letters 2006, 89, (16).

13. Weintraub, B.; Deng, Y. L.; Wang, Z. L., Journal of Physical Chemistry C 2007, 111, (28), 10162-10165.

14. Li, C.; Hong, G. S.; Wang, P. W.; Yu, D. P.; Qi, L. M., Chemistry of Materials 2009, 21, (5), 891-897.

15. Vayssieres, L., Advanced Materials 2003, 15, (5), 464-466.

16. Zhao, F.; Li, X.; Zheng, J.-G.; Yang, X.; Zhao, F.; Wong, K. S.; Wang, J.; Lin, W.; Wu, M.; Su, Q., Chemistry of Materials 2008, 20, (4), 1197-1199.

17. Hulteen, J. C.; Van Duyne, R. P., J. Vac. Sci. Technol. A 1995, 13, (3), 1553-1558. 
18. Haynes, C. L.; Van Duyne, R. P., Journal of Physical Chemistry B 2001, 105, (24), 5599-5611.

19. Deckman, H. W.; Dunsmuir, J. H., Journal of Vacuum Science \& Technology B 1983, 1, (4), $1109-1112$.

20. Haynes, C. L.; Van Duyne, R. P., Journal of Physical Chemistry B 2003, 107, (30), 7426-7433.

21. Zareie, H. M.; Morgan, S. W.; Moghaddam, M.; Maaroof, A. I.; Cortie, M. B.; Phillips, M. R., ACS Nano 2008, 2, (8), 1615-1619.

22. Fredriksson, H.; Alaverdyan, Y.; Dmitriev, A.; Langhammer, C.; Sutherland, D. S.; Zaech, M.; Kasemo, B., Advanced Materials 2007, 19, (23), 4297-+.

23. Pakizeh, T.; Abrishamian, M. S.; Granpayeh, N.; Dmitriev, A.; Kall, M., Optics Express 2006, $14,(18), 8240-8246$.

24. Jones, R. E.; Standley, C. L.; Maissel, L. I., Journal of Applied Physics 1967, 38, (12), 4656-\&.

25. Song, Q. M.; Wu, B. J.; Xie, B.; Huang, F.; Li, M.; Wang, H. Q.; Jiang, Y. S.; Song, Y. Z., Journal of Applied Physics 2009, 105, (4).

26. Jones, R. E.; Standley, C. L.; Maissel, L. I., Journal of Vacuum Science \& Technology 1966, 3, (5), 304-\&.

27. Jones, R. E.; Standley, C. L.; Maissel, L. I., Vacuum 1967, 17, (3), 165-\&.

28. Pliskin, W. A.; Davidse, P. D.; Lehman, H. S.; Maissel, L. I., Ibm Journal of Research and Development 1967, 11, (4), 461-\&.

29. Jones, R. E.; Winters, H. F.; Maissel, L. I., Journal of Vacuum Science \& Technology 1968, 5, (3), 84-\&.

30. Maissel, L. I.; Jones, R. E.; Standley, C. L., Ibm Journal of Research and Development 1970, 14, (2), 176-\&.

31. Koenig, H. R.; Maissel, L. I., Ibm Journal of Research and Development 1970, 14, (2), 168-\&.

32. Polykov, A. Y.; Smirnov, N. B.; Kozhukhova, E. A., Applied Physics Letters 2003, 83, (8), 1575-1577.

33. Dhananjay; Nagaraju, J.; Krupanidhi, S. B., Physica B 2007, 391, 344-349.

34. Coburn, J. W.; Taglauer, E.; Kay, E., Journal of Applied Physics 1974, 45, (4), 1779-1786.

35. Coburn, J. W.; Eckstein, E. W.; Kay, E., Journal of Applied Physics 1975, 46, (7), 2828-2830.

36. Tuyen, L. T. T.; Vinh, D. X.; Khoi, P. H.; Gerlach, G., Sensors and Actuators B-Chemical 2002, $84,(2-3), 226-230$.

37. Huang, M. H.; Wu, Y. Y.; Feick, H.; Tran, N.; Weber, E.; Yang, P. D., Advanced Materials 2001, 13, (2), 113-116.

38. Xu, S.; Adiga, N.; Ba, S.; Dasgupta, T.; Wu, C. F. J.; Wang, Z. L., ACS Nano 0, (0).

39. Ahsanulhaq, Q.; Kim, J. H.; Hahn, Y. B., Nanotechnology 2007, 18, (48). 
40. Sepulveda-Guzman, S.; Reeja-Jayan, B.; de la Rosa, E.; Torres-Castro, A.; Gonzalez-Gonzalez, V.; Jose-Yacaman, M., Materials Chemistry and Physics 2009, 115, (1), 172-178.

41. Cao, B. Q.; Cai, W. P.; Sun, F. Q.; Li, Y.; Lei, Y.; Zhang, L. D., Chemical Communications 2004, (14), 1604-1605.

42. Cui, M. L.; Wu, X. M.; Zhuge, L. J.; Meng, Y. D., Vacuum 2007, 81, (7), 899-903.

43. Li, Q. H.; Wan, Q.; Liang, Y. X.; Wang, T. H., Applied Physics Letters 2004, 84, (22), 45564558.

44. Ton-That, C.; Phillips, M. R.; Foley, M.; Moody, S. J.; Stampfl, A. P. J., Applied Physics Letters 2008, 92, (26).

45. Wang, X. J.; Vlasenko, L. S.; Pearton, S. J.; Chen, W. M.; Buyanova, I. A., Journal of Physics D-Applied Physics 2009, 42, (17).

Table of Contents (TOC)

Exploiting Zinc Oxide Re-Emission to Fabricate Periodic Arrays

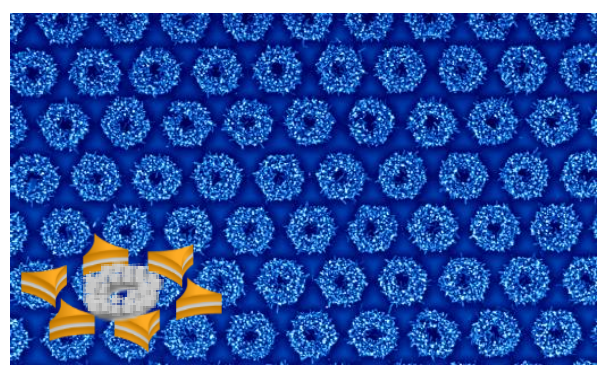

